\title{
Mobile Emergency Generator Pre-positioning and Real-time Allocation for Resilient Response to Natural Disasters
}

\author{
Shunbo Lei, Student Member, IEEE, Jianhui Wang, Senior Member, IEEE, \\ Chen Chen, Member, IEEE, and Yunhe Hou, Senior Member, IEEE
}

\begin{abstract}
Truck-mounted mobile emergency generators (MEGs) are critical flexibility resources of distribution systems (DSs) for resilient emergency response to natural disasters. However, they are currently under-utilized. For better utilization, this paper proposes dispatching MEGs as distributed generators in DSs to restore critical loads by forming multiple microgrids (MGs). As the travel time of MEGs on road networks (RNs) can greatly influence the outage duration of critical loads, a two-stage dispatch framework consisting of pre-positioning and real-time allocation is introduced, and the traffic issue is considered via the vehicle routing problem. Pre-positioning places MEGs in staging locations prior to a natural disaster, while real-time allocation sends MEGs from staging locations to restore critical loads by forming MGs in DSs after the natural disaster strikes. Specifically, with the objective of minimizing the expected outage duration of loads considering their priorities and demand sizes, pre-positioning is done via a scenario-based two-stage stochastic optimization problem, in which the first-stage pre-positioning decisions are evaluated by numbers of second-stage real-time allocation problems corresponding to considered scenarios of DS damage and RN damage/congestion. A scenario decomposition algorithm is applied to solve this problem. Illustrative cases demonstrate the effectiveness of the proposed dispatch scheme and algorithm.
\end{abstract}

Index Terms-Distribution system, microgrid, mobile emergency generator, resilience, stochastic optimization.

\section{NOMENCLATURE}

$\begin{array}{ll}\text { A. Indices: } \\ h, i, j, k & \text { Indices of DS nodes } \\ m & \text { Index of MEGs } \\ n & \text { Index of scenarios } \\ s & \text { Index of staging locations for pre-positioning }\end{array}$

This work was supported in part by National Natural Science Foundation of China (51277155), and Research Grant Council, Hong Kong SAR, under grant (ECS739713). J. Wang's work is supported by the U.S. Department of Energy (DOE)'s Office of Electricity Delivery and Energy Reliability.

S. Lei and Y. Hou are with the Department of Electrical and Electronic Engineering, the University of Hong Kong, Pokfulam, Hong Kong SAR, China. Y. Hou is also with the University of Hong Kong Shenzhen Institute of Research and Innovation, Shenzhen 518057, China (e-mail: leishunbo@eee.hku.hk; yhhou@eee.hku.hk).

J. Wang and C. Chen are with Argonne National Laboratory, Argonne, IL 60439 USA (e-mail: jianhui.wang@ anl.gov; morningchen@ anl.gov).
B. Sets:
B Set of all DS nodes, $i \in \mathbf{B}$
F, G Set of feeder root nodes, and set of candidate nodes for MEG connection, $k \in\{\mathbf{F}, \mathbf{G}\}$
M Set of MEGs, $m \in \mathbf{M}$
$\mathbf{N}$ Set of scenarios, $n \in \mathbf{N}$
$\mathbf{S} \quad$ Set of staging locations for pre-positioning, $s \in \mathbf{S}$
$\mathbf{L} \quad$ Set of distribution lines, $(i, j) \in \mathbf{L}$

C. Parameters:

$X_{s} \quad$ Allowed number of MEGs pre-positioned to staging location $s$

$t_{s k n} \quad$ Travel time of a MEG from staging location $s$ to node $k$ of DS under scenario $n$

$T_{\text {in }} \quad$ Estimated restoration time for the load at node $i$ under scenario $n$ by conventional restoration

$u_{n} \quad$ Probability or weight of scenario $n$

$p_{i}, q_{i} \quad$ Real and reactive power demand at node $i$

$w_{i} \quad$ Priority weight of power demand at node $i$

$r_{i j}, x_{i j} \quad$ Resistance and reactance of line $(i, j)$

$V_{0}, \varepsilon \quad$ Rated voltage and voltage deviation tolerance

$P_{m}^{\max }, Q_{m}^{\max }$ Maximum real and reactive power output of MEG $m$

$S_{i j}{ }^{\max } \quad$ Apparent power capacity of line $(i, j)$

$\theta_{k}(i) \quad$ Parent node of node $i$ regarding node $k$

$S_{i}{ }^{k} \quad$ Set of child nodes of node $i$ regarding node $k$

$\zeta_{k}(i, j) \quad$ Child node of line $(i, j)$ regarding node $k$

$\Pi \quad$ A sufficiently big number

D. Variables:

$x_{s m} \quad$ Binary, 1 if MEG $m$ is pre-positioned to staging location $s, 0$ otherwise

$y_{s m k n} \quad$ Binary, 1 if MEG $m$ is real-time allocated (sent) from staging location $s$ to node $k$ under scenario $n$, 0 otherwise

$z_{k n} \quad$ Binary, 1 if node $k$ is a feeder root node or a MEG is connected to it under scenario $n, 0$ otherwise

$v_{i k n} \quad$ Binary, 1 if node $i$ belongs to the MG powered by the power source at node $k$ under scenario $n, 0$ otherwise 


$\begin{array}{ll}c_{i j n} & \begin{array}{l}\text { Binary, } 1 \text { if line }(i, j) \text { is closed under scenario } n, 0 \\ \text { otherwise }\end{array} \\ l_{i n} & \begin{array}{l}\text { Binary, } 1 \text { if the switch of the load at node } i \text { is } \\ \text { closed under scenario } n, 0 \text { otherwise }\end{array} \\ \gamma_{i k n} & \begin{array}{l}\text { Binary, } 1 \text { if the load at node } i \text { is picked up by the } \\ \text { power source at node } k \text { under scenario } n, 0 \text { otherwise }\end{array} \\ P_{i n}^{k}, Q_{i n}^{k} \quad & \begin{array}{l}\text { Real and reactive in-flow power of node } i \\ \text { regarding the MG powered by the power source at }\end{array} \\ & \text { node } k \text { under scenario } n \\ V_{i n}^{k}, \delta_{i n}^{k} & \begin{array}{l}\text { Voltage and auxiliary voltage slack variable of } \\ \text { node } i \text { regarding the MG powered by the power } \\ \text { source at node } k \text { under scenario } n\end{array}\end{array}$

\section{INTRODUCTION}

$\mathrm{N}$ ATURAL disasters have been causing severe power outages in recent years. For example, in 2012, after Hurricane Sandy struck the East Coast of the U.S., approximately 8.35 million customers were reported without power [1]. Weather-related power outages have introduced tremendous economic loss and significant life risk, highlighting the importance of enhanced power grid resilience [2]. Rapid and effective response for electric service restoration is one of the critical requirements of a resilient power grid, as most recovery activities greatly depend on a reliable power supply [3]. However, a natural disaster can cause widespread and severe damage to power grids, leaving numerous customers without power for days, sometimes even for over a week. For faster restoration, resilient response strategies are critically necessary when communities are threatened by natural disasters.

Mobile emergency generators (MEGs) are critical flexibility resources for fast electric service restoration across distribution systems (DSs), especially when customers are without complete power access to the main grid, which is often the case after a natural disaster strikes [4],[5],[6]. MEGs are truck-mounted generators with the merits of mobility and large capacity (up to several MVA). They can be one of the most effective response resources when sustained damage leads to prolonged electric service outages in DSs. However, they are currently not well utilized. For example, before Hurricane Sandy struck, 400 industrial-size truck-mounted emergency response generators were prepared by the Federal Emergency Management Agency (FEMA), but only a fraction of them were providing power even three days after Sandy made landfall [7]. Efficient utilization of MEGs, i.e., an effective and fast response, is significantly hindered by several inter-related challenges. First, assessments are necessary to identify each outage area's needs for different types of MEGs. Currently, assessments are conducted after natural disasters strike and can take from hours to days. Second, reasonable matching between MEGs and unserved loads is required to ensure rational utilization of MEG resources. This matching can be quite difficult when multiple factors such as grid damage, operation modes, and load priorities are considered. Third, road network (RN) damage and congestion can prevent timely dispatch of MEGs, as natural disasters can lead to quite inefficient traffic, which is also a complex yet necessary consideration in MEG dispatch.

The literature contains little research on MEG utilization, i.e., dispatch, for resilient response to natural disasters. In [8], a detailed system design for MEGs using fuel cells is presented and studied experimentally. A MEG design based on an integrated controller for both single-phase and three-phase distribution line backup is provided and tested in [9]. Reference [10] studies risks of network protection provided by MEGs. Various designs of MEGs and their backup role during emergency situations in the British telecommunications network are briefly introduced in [11]. Telecommunications companies in Japan are also using MEGs to ensure telecommunications services, especially after an earthquake [12]. However, dispatch strategies are not presented in [11] or [12], and telecommunications networks are quite different from the power grid.

In [13] and [14], optimal MEG dispatch is studied. Reference [13] minimizes the sum of MEG investment and maintenance cost and customers' outage cost, via a genetic algorithm. Reference [14] first classifies and prioritizes loads according to multiple safety factors, and then minimizes load loss by a hierarchical dispatch strategy. However, the effectiveness of models or methods presented in [13] and [14] is quite limited. First, consequences of natural disasters (DS damage, RN damage/congestion, etc.) are not appropriately considered or modeled. Second, a detailed formulation for the operation of DSs is not included.

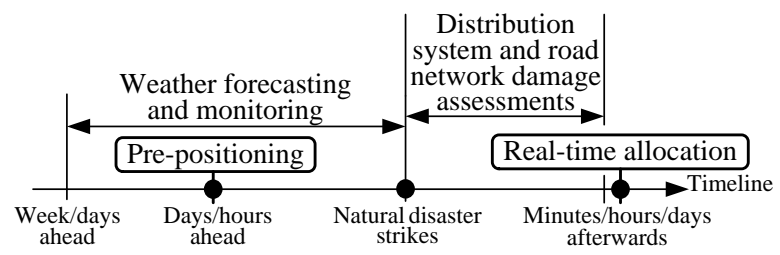

Fig. 1. Timing of pre-positioning and real-time allocation

In the present paper, it is proposed to dispatch MEGs as distributed generators (DGs) to some nodes of the DS to restore critical loads by forming multiple microgrids (MGs). In [4], a novel electric service restoration approach that forms MGs energized by existing DGs is proposed and studied. One of the significant differences is that the method in the present paper involves optimal placement of MEGs, while DGs are preinstalled resources in [4]. Note that the problem considered in the present work is also quite different from the optimal DG placement problem, which is generally studied assuming normal operation states for DSs, to reduce power loss, enhance voltage profile, improve service reliability, etc. [15]. Also, the present paper proposes to dispatch MEGs in a two-stage framework that comprises pre-positioning and real-time allocation. As shown in Fig. 1, prior to a natural disaster, according to damage forecasts for DSs and RNs, pre-positioning decisions are made via a scenario-based two-stage stochastic optimization problem to minimize the expected outage duration of loads considering their priorities and demand sizes. MEGs are then pre-positioned (i.e., placed) in staging locations for rapid real-time allocation in the future. After the natural disaster strikes, the actual real-time 
allocation is optimized. MEGs are then sent from staging locations to selected nodes in DSs to form MGs and pick up critical loads. A more detailed problem statement will be presented in Section II.

Major contributions are summarized as follows:

1) Dispatching MEGs as DGs in DSs to restore critical loads by forming multiple MGs is proposed and studied. This novel strategy is effective in matching MEGs with critical loads, thus achieving better capacity utilization of MEGs.

2) A two-stage framework to dispatch MEGs is introduced. With proactive pre-positioning and timely real-time allocation of MEGs, the outage duration of critical loads to be picked up by MEGs can be significantly reduced compared to current practice.

3) The pre-positioning and real-time allocation problems are properly modeled. The aforementioned challenges for MEG dispatch, i.e., proper assessment of needs for MEGs, reasonable matching between MEGs and critical loads, and appropriate consideration of the traffic issue, are accommodated by the present formulations. Specifically, the travel time of MEGs on RNs is optimized and obtained by solving the vehicle routing (VR) problem, and then considered in both the pre-positioning and real-time allocation problems, to minimize the outage duration of critical loads to be picked up by MEGs.

Thus, MEGs can be much better utilized for resilient response to natural disasters. The rest of this paper is organized as follows: Section II describes in detail the MEG dispatch problem and our proposed methodology for MEG dispatch. Sections III and IV present formulations of the prepositioning and real-time allocation problems, respectively. Section V briefly discusses their implementation and the applied algorithms. Illustrative cases are included in Section VI. Section VII briefly concludes the paper.

\section{PROBlem Statement}

\section{A. MEG Dispatch in Response to Natural Disasters}

Essentially, the MEG dispatch problem is to allocate MEGs in the power grid (normally, the DS) to restore critical loads under emergency situations. Generally, a MEG may serve a single location such as a hospital or a government building. However, after a natural disaster, MEGs will have to play a more important role than that. First, most critical and large loads, which are small in number, have backup power access to multiple feeders or self-installed emergency generators. And note that many MEGs will be in a state of readiness before a natural disaster. Thus, a considerable number of MEGs can be spared to serve small yet critical or less-critical loads, which are numerous. Second, as natural disasters often cause prolonged outages for many customers, MEGs can be vital power sources for them for days or even for over a week after a natural disaster strikes. Moreover, MEG dispatch in response to natural disasters has the following characteristics:

1) Natural disasters often cause a complete or partial loss of power supply to DSs from the main grid. Major reasons include transmission system outages, substation faults, broken feeders or laterals of DSs, etc. Timely electric service recovery of isolated outage regions by conventional restoration can be hindered. These are the major areas of interest for MEG dispatch. Nevertheless, areas that still have power access to the main grid should also be considered, as operational constraints may prohibit them from being fully restored.

2) Sustained DS element damage, the resulting prolonged power outages to customers, and $\mathrm{RN}$ damage/congestion are quite uncertain prior to a natural disaster. Assessments of these may be completed in minutes or hours, or even days, after the natural disaster strikes. Then, matching between MEGs and critical loads is conducted to guide MEG dispatch. Matching can be a quite difficult and complicated task, considering grid damage, DS operational constraints, requirements for resource utilization efficiency of MEGs, desired timely restoration, etc.

3) MEGs are truck-mounted emergency response generators that will have to travel on RNs to allocated locations. The outage duration of critical loads to be picked up by MEGs is greatly influenced by MEGs' travel time. Some critical loads may also require a MEG to arrive before their backup power runs out. Note that RNs can be vulnerable to natural disasters, too. Thus, the traffic issue is a critical factor in MEG dispatch. To reduce the outage duration of critical loads, proactive predispatch measures (pre-positioning, in this paper) are necessary, and VR of MEGs should be considered.

The current practice of MEG dispatch generally follows an ineffective pattern. First, although MEGs are prepared to be ready in advance, major dispatch efforts are conducted after natural disasters strike. However, proactive measures are preferred. Second, assessment of needs for MEGs and matching between MEGs and critical loads are not carried out rationally. After damage and outage assessments, sub-districts request MEGs on the basis of some statistical data, and then match them to loads on the basis of limited experience. Third, the traffic issue, a critical factor as previously noted, is not appropriately considered. Thus, MEGs are currently under-utilized.

\section{B. Proposed Operation Strategy: MG Formation with MEGs}

This paper proposes dispatching MEGs as DGs to some nodes of DSs. MEGs then restore critical loads by forming multiple MGs that operate in islanded mode. The objective is to minimize the outage duration of loads, considering their priorities and demand sizes. Major decision variables include the following:

- Allocating MEGs: to allocate each MEG to which candidate node in DSs

- Forming MGs powered by MEGs: to open or close each line to form MGs, and to pick up or not pick up each load Conditions and constraints to be considered include:

- Capacity differences of MEGs

- Priorities of critical loads

- DS element damage

- RN damage/congestion

- Radial topology requirements, operational constraints, etc. Note also that, constrained by equipment or firmware requirements, only a fraction of the nodes in DSs will be feasible as candidate nodes for MEG allocation and connection. Optimization models are presented in Sections III and IV; first, a two-stage framework for realizing this operation strategy is introduced in Section II.C. 


\section{Proposed Dispatch Method: A Two-stage Framework}

The proposed operation strategy for MG formation with MEGs is fulfilled by a proposed two-stage dispatch framework, comprising pre-positioning and real-time allocation.

In the first stage, i.e., prior to a natural disaster, prepositioning is conducted. Resource pre-positioning is a common proactive measure undertaken by electric utilities. Before a natural disaster, they allot resources (including repair crews and restoration equipment) across their staging locations to ensure the earliest possible response after the natural disaster strikes [16]. We determine that pre-positioning of MEGs, i.e., placing utilities' MEGs in staging locations for earliest future response, is also necessary for several interrelated reasons:

- The earliest possible electric service recovery is desired.

- MEGs' travel time to allocated places can be saved.

- The influence of RN damage/congestion can be reduced.

In the second stage, i.e., after the natural disaster strikes, real-time allocation is optimized. MEGs are sent from staging locations to allocated locations. Upon arrival, they are connected to the grid and form MGs to pick up critical loads.

\section{PRE-POSITIONING PROBLEM ForMULATION}

\section{A. Objective Function}

$$
\min \sum_{n \in \mathbf{N}} u_{n} \sum_{i \in \mathbf{B}} w_{i} p_{i}\left[\left(1-\sum_{k \in\{\mathbf{F}, \mathbf{G}\}} \gamma_{i k n}\right) T_{i n}+\sum_{k \in \mathbf{G}} \sum_{s \in \mathbf{S}} \sum_{m \in \mathbf{M}} \gamma_{i k n} y_{s m k n} t_{s k n}\right]
$$

The objective (1) is minimizing the expected outage duration of loads, considering their priorities and demand sizes. Specifically, all the terms in the square brackets in whole, i.e. $\left[\left(1-\sum_{k \in\{\mathbf{F}, \mathbf{G}\}} \gamma_{i k n}\right) T_{i n}+\sum_{k \in \mathbf{G}} \sum_{s \in \mathbf{S}} \sum_{m \in \mathbf{M}} \gamma_{i k n} y_{s m k n} t_{s k n}\right]$, represent outage duration of loads. The second term in the parentheses, i.e. $\sum_{k \in\{\mathbf{F}, \mathbf{G}\}} \gamma_{i k n}$, indicates whether the load at node $i$ is restored by a feeder root node or a MEG in scenario $n$. Firstly, if it equals to 0 , this load is not restored, and will experience an outage duration of the estimated restoration time $T_{i n}$. In this case, $\left(1-\sum_{k \in\{\mathbf{F}, \mathbf{G}\}} \gamma_{i k n}\right)$ equals to 1 , and $\sum_{k \in \mathbf{G}} \sum_{s \in \mathbf{S}} \sum_{m \in \mathbf{M}} \gamma_{i k n} y_{s m k n} t_{s k n}$ equals to 0 . Thus $T_{\text {in }}$ takes effect in objective (1) as the outage duration of this load. Secondly, if $\sum_{k \in\{\mathbf{F}, \mathbf{G}\}} \gamma_{i k n}$ equals to 1 , this load is restored by two possible power sources. One possibility is that it obtains power from a feeder root node. Its outage duration is considered as zero here since its restoration can be conducted immediately. In this case, both $\left(1-\sum_{k \in\{\mathbf{F}, \mathbf{G}\}} \gamma_{i k n}\right)$ and $\sum_{k \in \mathbf{G}} \sum_{s \in \mathbf{S}} \sum_{m \in \mathbf{M}} \gamma_{i k n} y_{s m k n} t_{s k n}$ equal to 0 , making the terms in the square brackets in whole equal to 0 . The other possibility is that this load is recovered by a MEG. The MEG's travel time on RNs is seen as its outage duration. In this case, $\left(1-\sum_{k \in\{\mathbf{F}, \mathbf{G}\}} \gamma_{i k n}\right)$ still equals to 0 . And, assuming that this load is picked up by a MEG at node $k$ and this MEG is from staging location $s$, then the term $\sum_{k \in \mathbf{G}} \sum_{s \in \mathbf{S}} \sum_{m \in \mathbf{M}} \gamma_{i k n} y_{s m k n} t_{s k n}$ equals to $t_{s k n}$. By using (1), pre-positioning decisions with optimal expected performance of future real-time allocations are derived. Note that (1) contains quadratic terms of two binary variables, $\gamma_{i k n} y_{s m k n}$. For linearization, we replace each of them by an auxiliary binary variable $\tau_{\text {smikn }}$ and add constraints (2) to achieve equivalent conversion:

$$
\tau_{s m i k n} \leq \gamma_{i k n}, \tau_{s m i k n} \leq y_{s m k n}, \tau_{s m i k n} \geq \gamma_{i k n}+y_{s m k n}-1
$$

\section{B. Constraints}

$$
\begin{aligned}
& \sum_{m \in \mathbf{M}} x_{s m} \leq X_{s}, \forall s \\
& \sum_{s \in \mathbf{S}} x_{s m}=1, \forall m \\
& \sum_{k \in \mathbf{G}} y_{s m k n} \leq x_{s m}, \forall s, \forall m, \forall n \\
& \sum_{s \in \mathbf{S}} \sum_{m \in \mathbf{M}} y_{s m k n} \leq 1, \forall k, \forall n \\
& z_{k n}=\sum_{s \in \mathbf{S}} \sum_{m \in \mathbf{M}} y_{s m k n}, \forall k \in \mathbf{G}, \forall n \\
& z_{k n}=1, \forall k \in \mathbf{F}, \forall n \\
& \sum_{k \in\{\mathbf{F}, \mathbf{G}\}} v_{i k n} \leq 1, \forall i, \forall n \\
& v_{i k n} \leq z_{k n}, \forall k, \forall i, \forall n \\
& v_{k k n} \geq z_{k n}, \forall k, \forall n \\
& v_{i k n} \leq v_{j k n}, j=\theta_{k}(i), \forall k, \forall i, \forall n \\
& c_{i j n}=\sum_{k \in\{\mathbf{F}, \mathbf{G}\}} v_{h k n}, h=\zeta_{k}(i, j), \forall(i, j), \forall n \\
& \gamma_{i k n}=v_{i k n} l_{i n}, \forall k, \forall i, \forall n \\
& \sum_{j \in S_{i}^{k}} P_{j n}^{k}=P_{i n}^{k}-\gamma_{i k n} p_{i}, \forall k, \forall i, \forall n \\
& \sum_{j \in S_{i}^{k}} Q_{j n}^{k}=Q_{i n}^{k}-\gamma_{i k n} q_{i}, \forall k, \forall i, \forall n \\
& 0 \leq P_{i n}^{k} \leq v_{i k n} \Pi, \forall k, \forall i \neq k, \forall n \\
& 0 \leq Q_{i n}^{k} \leq v_{i k n} \Pi, \forall k, \forall i \neq k, \forall n \\
& 0 \leq P_{k n}^{k} \leq \sum_{s \in \mathbf{S}} \sum_{m \in \mathbf{M}} y_{s m k n} P_{m}^{\max }, \forall k \in \mathbf{G}, \forall n \\
& 0 \leq Q_{k n}^{k} \leq \sum_{s \in \mathbf{S}} \sum_{m \in \mathbf{M}} y_{s m k n} Q_{m}^{\max }, \forall k \in \mathbf{G}, \forall n \\
& \left(P_{i n}^{k}\right)^{2}+\left(Q_{i n}^{k}\right)^{2} \leq\left(S_{i j}^{\max }\right)^{2}, j=\theta_{k}(i), \forall k, \forall i \neq k, \forall n \quad(6 \mathrm{~g}) \\
& V_{k n}^{k}=z_{k n} V_{R}, \forall k, \forall n \\
& V_{i n}^{k}=V_{j n}^{k}-\frac{r_{i j} \cdot P_{i n}^{k}+x_{i j} \cdot Q_{i n}^{k}}{V_{R}}-\delta_{i n}^{k}, j=\theta_{k}(i), \forall k, \forall i \neq k, \forall n \\
& 0 \leq V_{i n}^{k} \leq v_{i k n} V_{R}, \forall k, \forall i, \forall n \\
& 0 \leq \delta_{i n}^{k} \leq\left(1-v_{i k n}\right) V_{R}, \forall k, \forall i, \forall n \\
& \sum_{k \in\{\mathbf{F}, \mathbf{G}\}} v_{i k n}(1-\varepsilon) V_{0} \leq \sum_{k \in\{\mathbf{F}, \mathbf{G}\}} V_{i n}^{k} \leq \sum_{k \in\{\mathbf{F}, \mathbf{G}\}} v_{i k n}(1+\varepsilon) V_{0}, \forall i, \forall n \\
& c_{i j n}=0, \forall n, \forall(i, j) \in \mathbf{L O}_{n}
\end{aligned}
$$

The above constraints can be divided into five different groups as follows:

1) Pre-positioning constraints (3a) and (3b), to pre-position each MEG, and to avoid violating staging location capacity: The number of pre-positioned MEGs at each staging location 
is limited by its capacity as in (3a). Constraints (3b) ensure that each MEG is pre-positioned to exactly one of the staging locations.

2) Real-time allocation constraints (4a) and (4b), to send MEGs from staging locations: Constraints (4a) ensure that in each scenario, MEG $m$ is sent to one of the candidate nodes in the DS from staging location $s$ where it is pre-positioned. Constraints (4b) declare that at most one MEG is allocated to each candidate node.

3) DS topology reconfiguration constraints (5a)-(5h), to define feasible DS topologies, which satisfy the radial topology requirements and divide the DS into multiple MGs each with one power source: This and the next groups of constraints define the operational feasible set of related variables to realize the strategy of MG formation with MEGs. Only a brief explanation is presented here, owing to space limitations. Although the models have major differences, one can still refer to [4] for more details. Note that other than isolated outage regions, areas still having power access to the main grid are also modeled for the aforementioned reason. For statement simplicity, here we refer to the sub-grid powered by feeder root node $k$ or a MEG at node $k$ as MG $k$. In (5a) and (5b), auxiliary variables $z_{k n}$ indicate whether node $k$ has a power source (i.e. $z_{k n}=1$ if it is connected to a MEG or it is a feeder root node, $z_{k n}=0$ otherwise). Constraints (5c) ensure that each node belongs to at most one MG ( $v_{i k n}=1$ if node $i$ belongs to MG $k, v_{i k n}=0$ otherwise). Constraints (5d) prevent construction of a MG without a power source (if node $k$ does not have any power source, i.e. $z_{k n}=0$, then the formulation should not form the MG $k$, i.e. $v_{i k n}=0$ for all $i$ ). Constraints (5e) determine that each node with a power source belongs to the MG powered by itself (i.e., $v_{k k n}=1$ if $z_{k n}=1$ ). Constraints (5f) ensure that a node can belong to MG $k$ only if its parent node also belongs to MG $k$, because of the connectivity feature of a tree (in a radial DS network, each MG can be seen as a sub-tree network with the power source node being the root node). Equations $(5 \mathrm{~g})$ mean that a distribution line should be closed if its child node belongs to one of the MGs (conditioning that both node $i$ and $j$ belong to MG $k$, i.e. $v_{i k n}=v_{j k n}=1$, then the line $(i, j)$ also belongs to MG $k$, i.e. $c_{i j n}=1$ in the closed state; considering constraints (5f), this condition is equal to that the child node of the line $(i, j)$ belongs to MG $k$ ). The radial topology of DSs is preserved by constraints (5c)-(5g). Constraints (5h), which can be linearized in a similar manner to (2), guarantee that the load at node $i$ is picked up by MG $k$ only if node $i$ belongs to MG $k$ and the load switch is also closed.

4) DS operational constraints (6a)-(61), to constrain line flows, voltages and MEG power outputs: Equations (6a) and (6b) are the real and reactive power balance of each node, based on the DistFlow model [17], [18]. Constraints (6c) and (6d) ensure that the in-flow power of node $i$ regarding MG $k$ is zero if it does not belong to MG $k$ (i.e., $P_{i n}^{k}=Q_{i n}^{k}=0$ if $v_{i k n}=0$ ). Constraints (6e) and (6f) limit real and reactive power injection at a candidate node subject to the capacity of the connected MEG (if the $m$ th MEG is connected to node $k$, its power injections $P_{k n}^{k}$ and
$Q_{k n}^{k}$ should be less than its capacity $P_{m}^{m a x}$ and $\left.Q_{m}^{m a x}\right)$. Constraints (6g) are apparent power capacity constraints of lines, which can be linearized by techniques such as those given in [19]. Equations (6h) set voltages at nodes with a power source as the reference value $V_{R}=(1+\varepsilon) V_{0}$. Equations (6i) express relationships of voltages between connected nodes regarding MG $k$. Constraints (6j) ensure that the voltage value regarding MG $k$ is zero if a node does not belong to MG $k$ (i.e., $V_{i n}^{k}=0$ if $\left.v_{i k n}=0\right)$. Constraints $(6 \mathrm{k})$ define the range of voltage slack variables that guarantee the satisfaction of (6i). Constraints (61) set the voltage value range if a node belongs to one of the MGs.

5) DS damage scenario condition constraints (7), to give scenario conditions: With $\mathbf{L} \mathbf{O}_{n}$ as the set of damaged lines under scenario $n$, constraints (7) restrict them as inoperable in an open state.

Thus, the pre-positioning formulation is a scenario-based twostage stochastic optimization problem. The first-stage prepositioning decisions are evaluated by numbers of second-stage real-time allocation problems corresponding to the considered scenarios of DS damage and RN damage/congestion.

\section{REAL-TIME ALLOCATION PROBLEM FORMULATION}

Provided optimal pre-positioning decisions, i.e., determined $x_{s m}$, the actual real-time allocation of MEGs is conducted after the natural disaster strikes and damage assessments of DSs and $\mathrm{RNs}$ are finished. It is optimized via the following formulation, with only one scenario $n$ representing the reality resulting from the natural disaster:

$$
\begin{aligned}
& \min \sum_{i \in \mathbf{B}} w_{i} p_{i}\left[\left(1-\sum_{k \in\{\mathbf{F}, \mathbf{G}\}} \gamma_{i k n}\right) T_{i n}+\sum_{k \in \mathbf{G}} \sum_{s \in \mathbf{S}} \sum_{m \in \mathbf{M}} \tau_{s m i k n} t_{s k n}\right] \\
& \text { s.t. (2), (4a)-(4b), (5a)-(5h), (6a)-(61), (7) }
\end{aligned}
$$

Some other constraints can also be included, such as tolerable interruption duration $\bar{t}_{i}$ of a critical load at node $i$ :

$$
\begin{gathered}
\sum_{k \in \mathbf{G}} \gamma_{i k n} \geq 1 \\
\sum_{k \in \mathbf{G}} \sum_{s \in \mathbf{S}} \sum_{m \in \mathbf{M}} \gamma_{i k n} y_{s m k n} t_{s k n} \leq \bar{t}_{i}
\end{gathered}
$$

If the data of MEG installation time are available, they can be considered by simply adding to $t_{s k n}$ in (1) (8) and (9b). And capacity utilization rate (CUR) requirement $\underline{R}$ of MEGs:

$$
\sum_{i \in \mathbf{B}} \gamma_{i k n} p_{i} \geq \underline{R} \sum_{s \in \mathbf{S}} \sum_{m \in \mathbf{M}} y_{s m k n} P_{m}^{\max }, \forall k
$$

where we define the CUR of a MEG as the sum of real power loads served by it, divided by its real power capacity. With available MEG installation time data,

Next, several remarks regarding the formulations of both the pre-positioning and real-time allocation problems are provided:

Remark 1: regarding the selection of available MEGs. Currently we set capacities of available MEGs as parameters in our formulations. In some cases, dispatchers have to or are allowed to select a budgeted number of MEGs among different types. Our formulations can accommodate these cases by listing enough number of MEGs of each type and letting the optimizer to choose. A budget constraint limiting the amount 
of selected MEGs should also be added. In this way, the capacities of MEGs can be co-optimized with other decisions. Note that this may introduce extra computational burden. Experience-based heuristics may help to relieve it. For example, an initial solution of good quality may be set at first by the dispatchers based on experience.

Remark 2: regarding the number of MEGs in MGs. To maintain functionality of a MG with two or more generation units, their coordination has to be resolved [20] [21]. This can be a difficult issue not only for temporarily-install MEGs but also for pre-installed generators due to communication obstruction and the post-event degraded state of the system after natural disasters. Thus, although allowing two or more MEGs in a MG can be more advantageous in matching power with loads and improving voltage security, in this work we choose to form each MG with only one power source following [4]. For some types of MEGs equipped with required functional modules, parallel operation of 2 or 3 MEGs can be conducted by well-trained staff [12]. The premise is that they are connected to the same DS node. Our formulations can accommodate this special case by minor modifications. Specifically, constraints (4b) and (5a) should be modified as follows:

$$
\begin{gathered}
\sum_{s \in \mathbf{S}} \sum_{m \in \mathbf{M}} y_{s m k n} \leq 3, \forall k, \forall n \\
\frac{\sum_{s \in \mathbf{S}} \sum_{m \in \mathbf{M}} y_{s m k n}}{3} \leq z_{k n} \leq \sum_{s \in \mathbf{S}} \sum_{m \in \mathbf{M}} y_{s m k n}, \forall k \in \mathbf{G}, \forall n
\end{gathered}
$$

Remark 3: regarding the selection of candidate nodes for MEG connection. Generally they are selected based on: 1) Site requirements. Their locations should have appropriate space to install and operate a MEG, free of potential risks such as flooding [12]. 2) Access requirements. Their locations should be reachable by the truck-mounted MEGs and fuel trucks via the RNs [12]. 3) Facility requirements. A node with connection panel to interface with plug-terminated cables of MEGs is preferred [11]. An underground fuel tank at its location is also a plus [11] [12]. 4) Some other considerations. For example the noise. If necessary, dispatchers can further reduce the number of candidate nodes based on their preferences or other factors such as distance.

Remark 4: regarding the consideration of other power or flexibility resources. In the current formulations, feeder root nodes representing power from substations are also modelled besides MEGs. Other power or flexibility resources, including DGs and the measures of partial load restoration/curtailment, can also be considered. Specifically, DGs can be treated in the same way as feeder root nodes. In this way, DGs restore critical loads by forming MGs exactly as that in [4]. Partial load restoration/curtailment can be incorporated by adding a continuous variable for each load and some easy modifications of the formulations. We omit this consideration as it is not always practically feasible. More importantly, it can potentially lead to over-positive results of the CUR, thus over-evaluating our performance in matching MEGs and loads. The focus of this paper is designing a method to better utilize MEGs in response to natural disasters.

\section{IMPLEMENTATION AND ALGORITHM}

\section{A. Implementation Discussions}

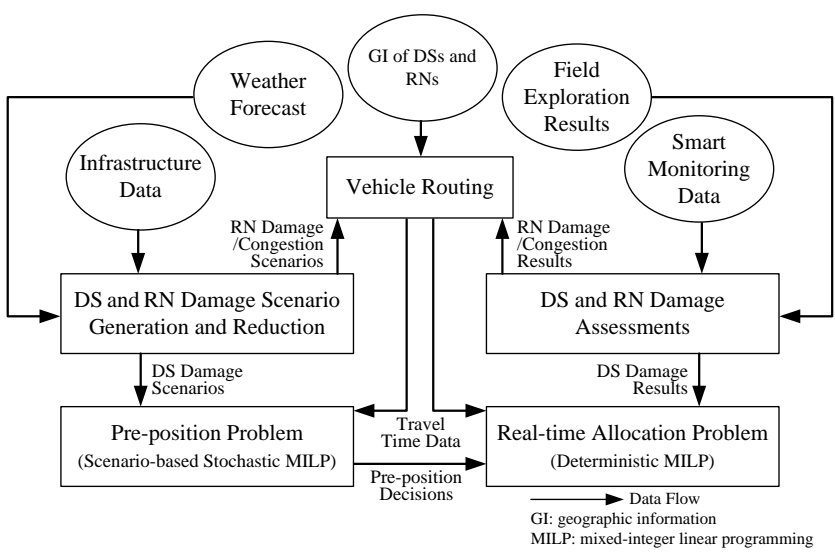

Fig. 2. Relationships between data sets and task modules

The foregoing Fig. 1 has shown the implementation timeline. Fig. 2, which is also self-explanatory, reveals the relationships among data sets and task modules that are necessary to implement MEG dispatch of pre-positioning and real-time allocation. On the basis of the framework depicted in Fig. 2, the proposed MEG dispatch methodology can also be reduced or modified to address the utility repair truck scheduling problem. Proactive pre-positioning and timely real-time response are also preferred in that problem. The detailed DS operation formulation is necessary to optimize the electric service recovery process, too. Note that the present paper focuses on the pre-positioning and real-time allocation modules for MEG dispatch.

Two selected issues related to implementation of the proposed MEG dispatch scheme are also discussed briefly as follows:

1) Scenario generation for natural disaster damages: On the one hand, statistical data fitting models use power grid data and environmental data to estimate outages and damages. Measurements of fitting goodness are also studied for evaluation. For example, models such as Bayesian Additive Regression Trees are assessed in estimating the number of damaged DS poles in [22]. On the other hand, simulationbased models make predictions based on physical mechanisms of damages. For example, in [23] the mechanism of localized high intensity wind damaging overhead line is studied. Interested readers may refer to [24], [25] and [26] for more detailed reviews. Thus, applying simulation-based or statistical models, scenario generation for natural disaster damages itself is a critical, challenging and active research topic. A potential topic is to consider graph-theoretic metrics here. In this work we generally assume that extensive expert experience or mature tools for scenario generation are available, and do not investigate too much detail on this topic since it is not the focus. Specifically, in Section VI, we generate scenarios following references [27] and [28], i.e. comparing failure probabilities of vulnerable components with a random variable uniformly distributed in the interval $(0,1)$. And we reduce scenarios by three rules. Firstly, prioritize scenarios that MEGs can restore more critical loads, since naturally it is of little meaning for the MEG dispatch problem to consider scenarios 
in which MEGs will not be quite helpful. Secondly, aggregate scenarios with the same real-time allocation solution as they tend to impact the pre-positioning decisions similarly. Thirdly, prioritize scenarios with higher probabilities of occurrence. The travel time of edges in RNs is assumed a lognormal distribution.

2) Coordination with the conventional DS restoration: The conceptual resilience curve in [29] is used here for more clear statements. In Fig. 3, $R$ is an index of system resilience level, $t_{M E G}$ the timing of MEGs participating into DS restoration.

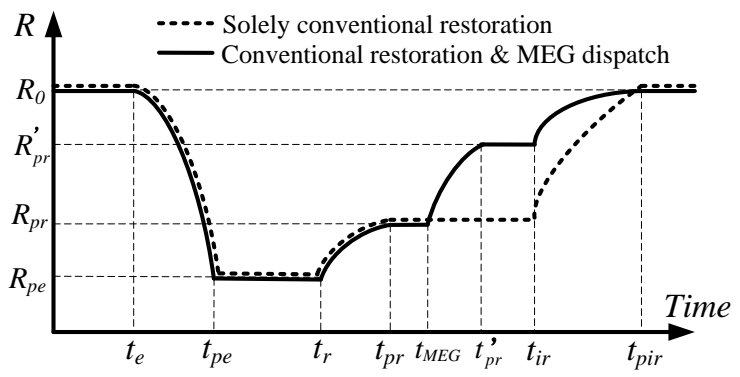

Fig. 3. A conceptual resilience curve associated with an event

Associated with an event, a DS has these states: event progress $t_{e} \sim t_{p e}$, post-event degraded state $t_{p e} \sim t_{r}$, restorative state $t_{r} \sim t_{p r}$, post-restoration state $t_{p r} \sim t_{i r}$ and infrastructure recovery $t_{i r} \sim t_{p i r}$. The time period $t_{r} \sim t_{i r}$ is the concern of conventional DS restoration strategies, which are generated by expert systems [30], multi-agent systems [31] and optimization [32], etc. However, they may be of limited effect for a DS struck by a natural disaster. That is, the enhancement from $R_{p e}$ to $R_{p r}$ is small. In this case MEGs are desirable resources to enhance the system resilience level from $R_{p r}$ to $R_{p r}^{\prime}$ in the postrestoration state. The concerned areas of MEG dispatch are mainly the isolated outage regions, and some areas that cannot be sufficiently restored by the surviving power access. Thus, although the concerned time period of MEG dispatch is the same as that of conventional DS restoration, their concerned outage areas are different with limited overlaps. These two kinds of restoration actions are related yet independent to some extent. Generally we can coordinate them in such a straightforward way: For outage areas sufficiently recovered by conventional restoration strategies, apply these strategies; for isolated outage areas without power sources, send MEGs and conduct MG formation after MEGs arrive; for outage areas insufficiently recovered by conventional restoration strategies, apply conventional strategies first, and then transfer to coordinated restoration strategies after MEGs arrive. Note that the coordinated strategies can be generated by solving our real-time allocation problem.

\section{B. Algorithm}

1) Dijkstra's shortest-path algorithm for the VR problem: As mentioned above, the traffic issue can influence optimal MEG dispatch decisions, as MEGs have to spend time traveling on RNs to allocated locations. Thus, a VR module is employed, which finds the shortest or fastest route from an origin to a destination via RNs. VR can be realized by Dijkstra's algorithm [33], the Floyd-Warshall algorithm [34], etc. We apply Dijkstra's algorithm here, as only routes from staging locations to candidate nodes are of interest. For each scenario, the travel time from each staging location to each candidate node is derived via the VR module. Then the data are used both in the pre-positioning and real-time allocation optimization problems. Thus, the traffic issue is considered when optimizing MEG dispatch to reduce the outage duration of critical loads to be picked up by MEGs. One might also consider MEGs' exploration role in damage assessment of DSs and RNs by assigning must-pass locations.

2) Scenario decomposition (SD) algorithm for prepositioning: The scenario-based two-stage stochastic optimization problem of pre-positioning has a block-diagonal structure. It can be recast in a compact form as follows:

$$
\min \left\{\sum_{n} f_{n}\left(\mathbf{x}, \mathbf{y}_{n}\right): \mathbf{x} \in \boldsymbol{\Lambda}, \mathbf{y}_{n} \in \mathbf{\Omega}_{n}\right\}
$$

where $\mathbf{x}, \mathbf{y}_{n}, f_{\mathrm{n}}\left(\mathbf{x}, \mathbf{y}_{n}\right), \boldsymbol{\Lambda}$ and $\boldsymbol{\Omega}_{n}$ denote first-stage prepositioning variables, second-stage real-time allocation variables under scenario $n$, second-stage objective function for scenario $n$, the feasible set of $\mathbf{x}$ defined by constraints (3a)(3b), and the feasible set of $\mathbf{y}_{n}$ defined by constraints (4a)-(4b), (5a)-(5h), (6a)-(6l) and (7), respectively. Generally, the SD algorithm is to transform (11) into (12):

$$
\min \left\{\sum_{n} f_{n}\left(\mathbf{x}_{n}, \mathbf{y}_{n}\right): \mathbf{x}_{n} \in \mathbf{\Lambda}, \mathbf{y}_{n} \in \mathbf{\Omega}_{n}, \sum_{n} \mathbf{A}_{n} \mathbf{x}_{n}=\mathbf{h}\right\}
$$

where the last constraint is the non-anticipativity constraints enforcing $\mathbf{x}_{l}=\mathbf{x}_{2}=\ldots=\mathbf{x}_{n}$. Thus the problem's block-diagonal structure can be taken advantage of. Implementation of the SD algorithm is described in Algorithm 1 [35].

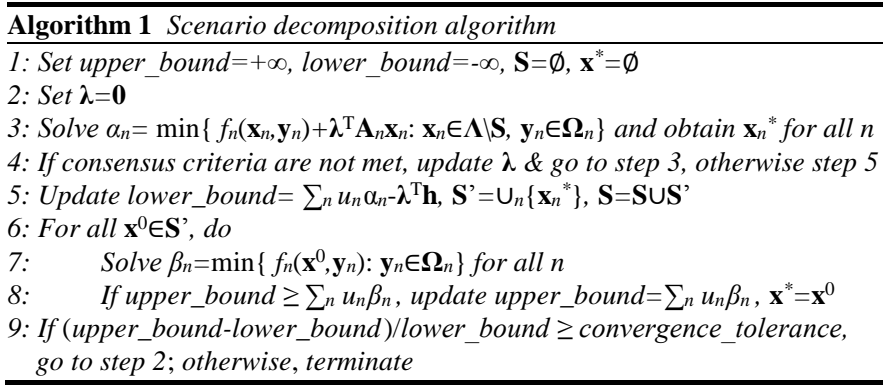

It is essentially a modified version of the progressive hedging algorithm [36], which can be classified as an augmented Lagrangian relaxation algorithm. Specifically, $f_{n}\left(\mathbf{x}_{n}, \mathbf{y}_{n}\right)+\lambda^{\mathrm{T}} \mathbf{A}_{n} \mathbf{x}_{n}$, i.e. the objective function in step 3, can be rewritten in a more detailed form as follows:

$$
\min _{\mathbf{x}_{n}, \mathbf{y}_{n}} \sum_{l}\left[\boldsymbol{\mu}(l)+\lambda(l)-\rho \overline{\mathbf{x}}_{n}(l)+0.5 \rho\right] \mathbf{x}_{n}(l)+\vartheta(l) \mathbf{y}_{n}(l)
$$

where $\overline{\mathbf{x}}_{n}$ denotes the weighted average of $\mathbf{x}_{n}$ over all scenarios $n \in \mathbf{N}$ in the previous iteration; $\boldsymbol{\mu}$ and $\boldsymbol{\vartheta}$ are cost coefficient vectors, i.e. problem parameters; $(\bullet)(l)$ denotes the $l$ th element of the vector $(\bullet) ; \rho$ is an algorithm parameter set as 10000 in this work. Note that, other than fixed $\rho$ strategies, there exist variable $\rho$ strategies. Interested readers can refer to [37], [38] and [39], which discuss both of them. And, $\lambda$ is updated by:

$$
\lambda(l) \leftarrow \lambda(l)+\rho\left[\mathbf{x}_{n}(l)-\overline{\mathbf{x}}_{n}(l)\right]
$$


which is a generally applied updating rule. And, the consensus criterion in step 4 is set as follows:

$$
t d=\sum_{l, n} \frac{\left|\mathbf{x}_{n}(l)-\overline{\mathbf{x}}_{n}(l)\right|}{|\mathbf{N}|} \leq \underline{t d}
$$

where $t d$ is defined as the average per-scenario deviation from the average; $\underline{t d}$ denotes a threshold set as 0.5 ; and $|\mathbf{N}|$ is the number of scenarios. The consensus criteria can also be set based on other metrics such as normalized average perscenario deviation from the average [37] and overall cost discrepancy [38]. As for the actual real-time allocation optimization problem to be solved after the natural disaster strikes, in our cases it can be directly solved by a solver like Gurobi with quite acceptable efficiency.

\section{ILLUSTRATIVE CASES}

\section{A. Test System Introduction}

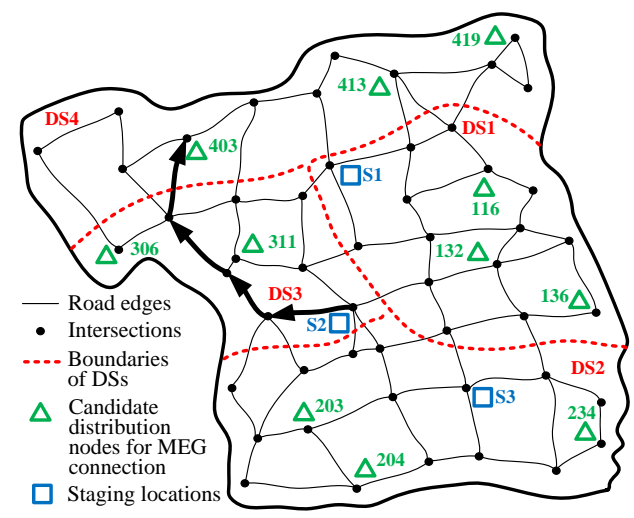

Fig. 4. Geographic information for RNs, DSs, staging locations, and candidate distribution nodes for MEG connection

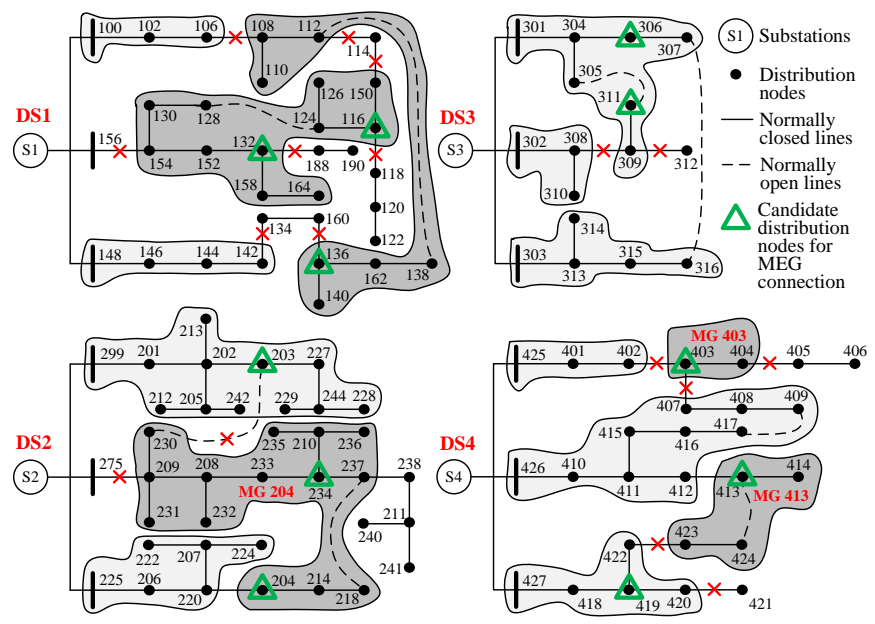

Fig. 5. Topology of DSs (DS1: a modified IEEE 34-node DS [40]; DS2: a modified IEEE 37-node DS [41]; DS3: a modified 16-node DS [42]; DS4: a modified 27-node DS [43]) and a demonstration case

Fig. 4 and Fig. 5 show the test system used for illustration. As MEG dispatch often involves an area with multiple DSs, a geographic district with four DSs in four sub-districts is considered. The RNs shown in Fig. 4 have 51 intersections and
82 edges. The DSs in Fig. 5 have 114 nodes in total. Loads and their priority weights are randomly generated. Several nodes in DSs are picked as candidate nodes for MEG connection. Assume that in this area, the utility has three staging locations, each of which can accommodate two MEGs, and has the MEG resources listed in Table I. A hurricane is considered, with high wind as one of the major influential factors. Damage scenarios for DSs are generated using lines' failure probabilities based on the fragility curve. Lognormal distribution is assumed for the travel time on each road edge in RNs among scenarios.

TABLE I

CAPACITIES OF AvaILABLE MEGs

\begin{tabular}{c|c|c}
\hline$m$ & $P_{m}^{\max }(\mathrm{kW})$ & $Q_{m}^{\max }(\mathrm{kVar})$ \\
\hline 1 & 400 & 300 \\
\hline 2 & 800 & 600 \\
\hline 3 & 1000 & 800 \\
\hline 4 & 1600 & 1200 \\
\hline 5 & 2000 & 1600 \\
\hline
\end{tabular}

\section{B. Demonstration of the Proposed MEG Dispatch Method}

In this sub-section, we demonstrate how the proposed MEG dispatch method, i.e., the two-stage framework of prepositioning and real-time allocation, is applied step by step:

1) Prior to the hurricane, with appropriately generated and selected scenarios, the pre-positioning problem is solved. It seeks pre-positioning of MEGs with optimal expected performance of future real-time allocations. Twenty scenarios are included here. Pre-positioning decisions for the studied case are shown in the second column of Table II.

TABLE II

A Demonstration CASE For the Proposed MEG Dispatch Method

\begin{tabular}{c|ccc}
\hline $\begin{array}{c}\text { Staging } \\
\text { locations }\end{array}$ & $\begin{array}{c}\text { Pre-positioning } \\
\text { decisions }\left(x_{s m}\right)\end{array}$ & $\begin{array}{c}\text { Real-time allocation } \\
\text { decisions }\left(y_{s m k n}\right)\end{array}$ \\
\hline \multirow{2}{*}{ S1 } & MEG 3 & $\rightarrow \rightarrow$ & Allocated to node 413 \\
& MEG 4 & $\rightarrow \rightarrow$ & Allocated to node 116 \\
\hline S2 & MEG 1 & $\rightarrow \rightarrow$ & Allocated to node 403 \\
\hline S3 & MEG 2 & $\rightarrow \rightarrow$ & Allocated to node 136 \\
\hline
\end{tabular}

2) After the hurricane strikes, assessments of DS damage and $\mathrm{RN}$ status are performed. Then the real-time allocation problem is solved for the resulting reality scenario. Assume that lines with a cross in Fig. 5 are damaged by the hurricane. Real-time allocation decisions are listed in the third column of Table II.

3) MEGs are then sent from staging locations to allocated nodes in DSs. Their travel routes on $\mathrm{RNs}$ are already determined in step 2 when using the VR module to compute necessary parameters (travel time $t_{s k n}$ ) for the real-time allocation problem. For example, MEG 1 at staging location S2 is assigned to node 403. According to the VR module, it travels on the arrowed bold edges in Fig. 4, and its travel time will be about 21 minutes. If updated RN status is available, a new route can also be generated.

4) Upon MEGs' arrivals at assigned locations, they are connected to DSs, and form MGs to restore critical loads. The 
formation of MGs is already decided in step 2 when solving the real-time allocation problem. In the assumed resulting reality scenario, the MG formation results are depicted in Fig. 5. Each more deeply shadowed area is an operating $\mathrm{MG}$ powered by a MEG. The MGs 204 and 413 marked in Fig. 5 explain the reason for including areas that still have power access to the main grid in formulations. Although loads in $\mathrm{MG}$ 204 and MG 413 can reach a feeder root node via undamaged lines, they cannot be fully restored because of operational constraints such as line flow limits. Therefore, MEGs are connected for better restoration. Note that some loads are still unserved. Complete electric service recovery relies on further efforts on conventional repair and restoration.

\section{Capacity Utilization of MEGs}

After natural disasters strike, the number of MEGs generally will be insufficient to meet demands on them. It is desired that MEGs be fully utilized to restore a maximal amount of critical load. Thus, the matching between MEGs and critical loads is important, as mentioned above. In this sub-section, the capacity utilization of MEGs is investigated. The evaluation process is to do simulations by solving real-time allocation problems for different scenarios based on fixed pre-positioning decisions, and then summarize the simulation results.

We use the foregoing CUR as an evaluation index here. If MEG $m$ is connected to node $k$ in scenario $n$, its CUR will be

$$
C U R=\left(\sum_{i \in \mathbf{B}} \gamma_{i k n} p_{i} / P_{m}^{\max }\right) \times 100 \%
$$

Based on pre-positioning decisions discussed in Section VI.B, we conduct 500 rounds of simulations of the real-time allocation. Statistics for the CUR of each MEG with different capacities are listed in the second column of Table III. With an average CUR of $67.43 \%$, the performance of the real-time allocation in matching MEGs with critical loads is acceptable. Actually, when using (8) as the objective function in real-time allocation problems, partial capacity of MEGs is sacrificed to achieve fast restoration of critical loads with high priorities. Here, we further investigate the CUR performance of a modified version of the real-time allocation problem. That is, the objective function (8) in the real-time allocation problem is replaced by the following one, which drops considerations of load priorities and travel time of MEGs on RNs:

$$
\max \sum_{i \in \mathbf{B}} \sum_{k \in\{\mathbf{F}, \mathbf{G}\}} \gamma_{i k n} p_{i}
$$

Again, 500 rounds of real-time allocation simulations are conducted. The statistics for the CUR are listed in the fourth column of Table III. As shown, the performance of capacity utilization of MEGs is now even better. Thus, one may alter settings of the objective function according to one's preference. In fact, whether using (8) or (17) as the objective, generally the proposed real-time allocation model can rationally match MEGs with loads as long as the topology of DSs allows. As indicated by the third and fifth columns of Table III, among a major fraction of simulated cases, the CUR of different types of MEGs reaches a level above $85 \%$. Note, however, that among the other simulated cases, damage to DSs resulting from the hurricane may force some of the MEGs to serve small amounts of loads in some small isolated outage areas, such as the MG 403 shown in Fig. 5.

TABLE III

SiMULATION STATISTICS FOR CAPACITY UTILIZATION RATE

\begin{tabular}{c|c|c|c|c}
\hline $\begin{array}{c}- \\
\text { MEG No. } \\
m\end{array}$ & \multicolumn{2}{|c|}{ Using obj. (8) } & \multicolumn{2}{c}{ Using obj. (17) } \\
\cline { 2 - 5 } & $\begin{array}{c}\text { Capacity } \\
\text { utilization rate }\end{array}$ & $\begin{array}{c}\text { Times when } \\
\text { CUR } \geq 85 \%\end{array}$ & $\begin{array}{c}\text { Capacity } \\
\text { utilization rate }\end{array}$ & $\begin{array}{c}\text { Times when } \\
\text { CUR } \geq 85 \%\end{array}$ \\
\hline 1 & $73.03 \%$ & 221 & $74.58 \%$ & 228 \\
\hline 2 & $64.13 \%$ & 97 & $80.87 \%$ & 252 \\
\hline 3 & $75.93 \%$ & 242 & $87.28 \%$ & 323 \\
\hline 4 & $63.65 \%$ & 149 & $71.36 \%$ & 221 \\
\hline 5 & $60.41 \%$ & 126 & $72.14 \%$ & 179 \\
\hline Average & $67.43 \%$ & 167 & $77.25 \%$ & 240.6 \\
\hline
\end{tabular}

Actually, saving $25 \sim 35 \%$ of MEGs' capacities as reserves is reasonable to accommodate fluctuation of loads, and helps to restore adjacent loads after some DS elements are quickly repaired. For example, in Fig. 5, after the line $(404,405)$ is repaired quickly, the loads at nodes 405 and 406 can be restored by the MEG at node 403. Electric service recovery for these nodes will not have to wait for repair and restoration of main grids and feeders, which may take much longer after a natural disaster.

Case studies in this sub-section validate the capability of the proposed strategy and formulations to match MEGs and loads, thus achieving better utilization of MEGs. First, the strategy of MG formation with MEGs can avoid low capacity utilization of MEGs. Secondly, their allocations and load pickup via MG formation are explicitly co-optimized by our formulations. Various constraints and conditions, which are major challenges for the current MEG dispatch practice, are appropriately included.

\section{Importance of Considering Traffic Issue and Pre-positioning}

As summarized in Table IV, real-time allocation is simulated 100 times under different settings for demonstration purposes. First, we show that to mitigate influence of the traffic issue on future real-time allocation, it is necessary to conduct proactive pre-positioning.

TABLE IV

SimUlation Statistics FOR LOAD RESTORATION AND MEG TRAVEL TiME

\begin{tabular}{c|c|c|c}
\hline & $\begin{array}{c}\text { Average amount of load } \\
\text { restored by MEGs }\end{array}$ & $\begin{array}{c}\text { Average travel time to } \\
\text { assigned locations }\end{array}$ \\
\hline Non-optimal pre-positioning & $3866.8 \mathrm{~kW}$ & 28.26 minutes \\
\hline $\begin{array}{l}\text { Optimal pre- } \\
\text { positioning }\end{array}$ & Using obj. (8) & $3910.9 \mathrm{~kW}$ & 21.58 minutes \\
\cline { 2 - 4 } & Using obj. (18) & $4082.5 \mathrm{~kW}$ & 35.05 minutes \\
\hline
\end{tabular}

As indicated by the third row of Table IV, with optimal prepositioning, the time spent by MEGs on RNs with real-time allocation is short. Once assessments of DGs and RNs are fully or partly completed after the hurricane, optimal real-time allocation decisions can be derived. MEGs are then sent to assigned locations and will arrive soon to restore critical loads. If pre-positioning is not performed, certainly one can still use the same real-time allocation decisions to restore the same number of critical loads. However, it can take much longer for MEGs to travel on RNs from other distant regions to allocated 
locations. When some administrative processes are included, outage durations for loads to be restored by MEGs can be hours or even days longer.

Moreover, rather than merely random pre-positioning, optimal pre-positioning is desired. The second row of Table IV shows statistics for real-time allocation simulations when randomly decided, non-optimal pre-positioning is assumed. Compared to real-time allocation simulations based on optimal pre-positioning, the amount of restored load is slightly less, and the travel time of MEGs on RNs is $30.95 \%$ longer.

Second, we show the importance of explicitly considering the traffic issue in real-time allocation. We experimentally alter the objective of real-time allocation to be the following one:

$$
\min \sum_{i \in \mathbf{B}} w_{i} p_{i}\left(1-\sum_{k \in\{\mathbf{F}, \mathbf{G}\}} \gamma_{i k n}\right) T_{i n}
$$

i.e., we drop the consideration of traffic issues. As indicated by the last row of Table IV, based on optimal pre-positioning, although the amount of restored load is slightly higher, this change leads to a longer outage duration of loads to be restored by MEGs.

Simulations demonstrate the importance of considering traffic issues and applying pre-positioning. Solving the VR problem by methods such as Dijkstra's algorithm, and then using related parameters in both pre-positioning and real-time allocation, are effective in reducing the outage duration of critical loads to be picked up by MEGs. In a practical system, the effectiveness can be more significant. To sum up, first, if proactive pre-positioning is not conducted, the outage duration of some critical loads can be much longer. Second, optimal pre-positioning does lead to better performance of real-time allocation than non-optimal pre-positioning. And third, to achieve the intended fast restoration, explicit consideration of traffic issues in performing real-time allocation is necessary.

\section{E. Computational Efficiency}

In this paper, mixed-integer linear programming problems are solved by a computer with an Intel $15-4278 \mathrm{U}$ processor and $8 \mathrm{~GB}$ of memory using Gurobi 6.0.4 with the default setting. The convergence tolerance of Algorithm 1 is set as $0.5 \%$.

As for the pre-positioning problem, we consider 20 scenarios. Applying the SD algorithm, the problem can be solved within 10.04 minutes in 8.2 iterations on average. The computational efficiency is acceptable, since pre-positioning is conducted as much as days in advance. Table $\mathrm{V}$ further compares the computation time using the SD algorithm to the solution time directly using Gurobi 6.0.4 (here, MIPGap set as $0.5 \%$ for fair comparison) with different numbers of scenarios:

TABLE V

Computation Time Comparison of Pre-Positioning Problem (minutes)

\begin{tabular}{c|c|c|c|c|c}
\hline \multirow{2}{*}{$\begin{array}{c}\text { Solution } \\
\text { Methods }\end{array}$} & \multicolumn{5}{|c}{ Scenario Number } \\
\cline { 2 - 6 } & 10 & 20 & 30 & 40 & 50 \\
\hline Gurobi 6.0.4 & 8.38 & 20.24 & 54.71 & 86.62 & 134.12 \\
\hline SD algorithm & 4.61 & 10.04 & 35.73 & 62.16 & 101.22 \\
\hline
\end{tabular}

Application of the SD algorithm generally reduces the computation time. However, with increasing scenario number, the computational efficiency improvement becomes less significant. Since a major advantage of Algorithm 1 is that it can be easily implemented in a distributed framework of parallel computing [35] [37], faster computation can be achieved by doing so. Note that decision makers can set the convergence tolerance according to the situations such as scenario number, computation framework (serial or parallel) and computation environment, etc. We set it as $0.5 \%$ here so that all pre-positioning problems for this section can be solved within a reasonable length of time, and it takes much longer time to further reduce the optimality gap. The choice of scenario number is also an important issue. Less scenarios are desired to achieve better computational efficiency, while more scenarios improve the quality of the optimal solution. Decision makers should also dependently decide the scenario number according to the situations. Firstly, one can consider increasing the scenario number if the optimal solution is not consistent. Secondly, running simulations to evaluate solutions can be another choice if increasing scenario number is not an option.

As for the real-time allocation problem, mostly it is solved rapidly within half a second. The computational efficiency is also acceptable.

\section{CONCLUSIONS}

Natural disasters often cause prolonged outages for many customers because of sustained damage of DS elements. MEGs are valuable flexibility resources for fast recovery of this electric service. This paper proposes to dispatch MEGs as DGs to restore critical loads by MG formation. This approach is realized by a two-stage dispatch framework with prepositioning and real-time allocation. Illustrative cases validate the effectiveness of the proposed MEG dispatch method. Explicitly considering the traffic issue and proactively executing pre-positioning can reduce the outage duration of critical loads. Rational matching between MEGs and loads is also achieved in real-time allocation. In summary, our proposed method for MEG dispatch is effective for reducing the outage scale and duration of critical loads after natural disasters strike. Thus, compared to the current practice, MEGs can be better utilized for resilient emergency response to natural disasters.

Some future research topics are worth investigating. Firstly, a robust real-time allocation strategy against uncertainties is desired to achieve fast response. Currently we are using perfect information for the real-time allocation problem. That is, we assume that damage assessments can be finished quickly to inform the system status. However, in some cases complete assessments of DS and RN damages can be quite timeconsuming. Thus, real-time allocation of MEGs based on imperfect information should be studies, especially for intended applications. Secondly, multi-period dynamic dispatch of MEGs can be co-optimized with the sequence to repair damaged elements in DSs. After actions including the conventional DS restoration and resilient response of MEGs, the DS stays in the post-restoration state for some time, and will start the infrastructure recovery process [29]. Damaged components will be repaired and the system will be restored dynamically. We will have to study how MEGs should 
respond dynamically in this infrastructure recovery process to reduce load outage. This problem is also important for intended applications of MEGs. Third, combined with the optimal repair sequence problem, the proposed MEG dispatch methodology can be reduced or modified for utility repair truck scheduling.

\section{REFERENCES}

[1] NERC, "Hurricane Sandy Event Analysis Report," 2014 [Online]. Available: http://www.nerc.com/pa/rrm/ea/Oct2012HurricanSandyEvnt AnlyssRprtDL/Hurricane_Sandy_EAR_20140312_Final.pdf

[2] R. J. Campbell, "Weather-related power outages and electric system resiliency," Congr. Res. Serv., Washington, DC, USA, Tech. Rep. R42696, 2012. [Online]. Available: http://www.fas.org/sgp/ crs/misc/R42696.pdf

[3] EPRI, "Enhancing Distribution Resiliency: Opportunities for Applying Innovative Technologies," 2013 [Online]. Available: http://www.epri. com/abstracts/Pages/ProductAbstract.aspx?ProductId=0000000000102 6889

[4] C. Chen, J. Wang, F. Qiu, and D. Zhao, "Resilient distribution system by microgrids formation after natural disasters," IEEE Trans. Smart Grid, vol. 7, no. 2, pp. 958-966, Mar. 2016.

[5] FEMA, "Mitigation assessment team report: Hurricane Sandy in New Jersey and New York," 2013 [Online]. Available: http://www.fema.gov /media-library/assets/documents/85922

[6] The GridWise Alliance, "Improving electric grid reliability and resilience: Lessons learned from Superstorm Sandy and other extreme events," 2013 [Online]. Available: http://www.gridwise.org/ documents/ImprovingElectricGridReliabilityandResilience_6_6_13web FINAL.pdf

[7] D. Barrett, "Few big FEMA generators humming," 2012 [Online]. Available: http://www.wsj.com/articles/SB100014240529702047071 04578093192471666514

[8] C. Yin, J. Wang, and T.-C. Ma, "System design of mobile emergency power plant based on fuel cells," in Proc. 2011 Int. Conf. on Electr. Inf. and Control Eng., Apr. 2011, pp. 5419-5422.

[9] C. Y. Jeong, J. G. Cho, J. W. Baek, and D. W. Yoo, "A new integrated controller based $100 \mathrm{kVA}$ mobile engine generator for single/three phase distribution line backup," in Proc. IEEE 15th Appl. Power Electron. Conf. and Expo., 2000, vol. 2, pp. 902-907.

[10] F. T. Dai, "Risks of network protection for mobile generator applications," in Proc. IET 9th Int. Conf. on Develop. in Power Syst. Protection, Mar. 2008, pp. 681-686.

[11] C. R. Nightingale, "The design of mobile engine driven generating sets and their role in the British telecommunications network," in Proc. 5th Int. Telecom. Energy Conf., Oct. 1983, pp. 144-150.

[12] S. Iwai, T. Kono, M. Hashiwaki, and Y. Kawagoe, "Use of mobile engine generators as source of back-up power," in Proc. IEEE 31st Int. Telecom. Energy Conf., Oct. 2009, pp. 1-6.

[13] L. Zhou, M. Fan, and Z. Zhang, "A study on the optimal allocation of emergency power supplies in urban electric network," in Proc. 20th Int. Conf. and Exhib. on Electr. Distrib., June 2009, pp. 1-4.

[14] J. Shang, X. Sheng, J. Zhang, and W. Zhao, "The optimized allocation of mobile emergency generator based on the loads importance," in Proc. 2009 Asia-Pacific Power and Energy Eng. Conf., Mar. 2009, pp. 1-4.

[15] P. S. Georgilakis and N. D. Hatziargyriou, "Optimal distributed generation placement in power distribution networks: Models, methods, and future research," IEEE Trans. Power Syst., vol. 28, no. 3, pp. 34203428, Aug. 2013.

[16] S. D. Whipple, "Predictive storm modelling and optimizing crew response to improve storm response operations," M. B. A. and M. Sc. Dissertation, MIT, Cambridge, MA, 2014.

[17] M. E. Baran and F. F. Wu, "Network reconfiguration in distribution systems for loss reduction and load balancing," IEEE Trans. Power Del., vol. 4, no. 2, pp. 1401-1407, Apr. 1989.

[18] H.-G. Yeh , D. F. Gayme, and S. H. Low, "Adaptive VAR Control for Distribution Circuits With Photovoltaic Generators," IEEE Trans. Power Syst., vol. 27, no. 3, pp. 1656-1663, Aug. 2012.

[19] X. Chen, W. Wu, and B. Zhang, "Robust restoration method for active distribution networks," IEEE Trans. Power Syst., in press.
[20] H. Qi, X. Wang, L. Tolbert, F. Li, F. Peng, P. Ning, and M. Amin, “A resilient real-time system design for a secure and reconfigurable power grid," IEEE Trans. Smart Grid, vol. 2, no. 4, pp. 770-781, Dec. 2011.

[21] F. Katiraei and M. R. Iravani, "Power management strategies for a microgrid with multiple distributed generation units," IEEE Trans. Power Syst., vol. 21, no. 4, pp. 1821-1831, Nov. 2006.

[22] S. D. Guikema, S. M. Quiring, and S.-R. Han, "Prestorm estimation of hurricane damage to electric power distribution systems," Risk Anal., vol. 30, no. 12, pp. 1744-1752, Dec. 2010

[23] G. Mcclure, S. Langlois, and J. Rogier, "Understanding how overhead lines respond to localized high intensity wind storms," in Proc. Struct. Congr., Vancouver, BC, Canada, 2008 [Online]. Available: http://ascelibrary.org /doi/abs/10.1061/41016(314)192

[24] S.-R. Han, "Estimating hurricane outage and damage risk in power distribution systems," Ph.D. dissertation, Texas A\&M Univ., College Station, TX, USA, 2008.

[25] S. D. Whipple, "Predictive storm modelling and optimizing crew response to improve storm response operations," M. B. A. and M. Sc. Dissertation, MIT, Cambridge, MA, 2014.

[26] Y. Wang, C. Chen, J. Wang, and R. Baldick, "Research on Resilience of Power Systems Under Natural Disasters-A Review," IEEE Trans. Power Syst., vol. 31, no. 2, pp. 1604-1613, May 2015.

[27] M. Ouyang and L. Dueñas-Osorio, "Time-dependent resilience assessment and improvement of urban infrastructure systems," Chaos, vol. 22, no. 3, 2012, Art. no. 033122.

[28] J. Winkler, L. Dueñas-Osorio, R. Stein, and D. Subramanian, "Performance assessment of topologically diverse power systems subjected to hurricane events," Rel. Eng. Syst. Safety, vol. 95, no. 4, pp. 323-336, 2010.

[29] M. Panteli and P. Mancarella, "The grid: Stronger, bigger, smarter?" IEEE Power \& Energy Mag., vol. 13. no. 3, pp. 58-66, May 2015.

[30] C. C. Liu, S. J. Lee, and S. S. Venkata, "An expert system operational aid for restoration and loss reduction of distribution systems," IEEE Trans. Power Syst., vol. 3, no. 2, pp. 619-626, May 1988

[31] C. P. Nguyen and A. J. Flueck, "Agent based restoration with distributed energy storage support in smart grids," IEEE Trans. Smart Grid, vol. 3, no. 2, pp. 1029-1038, Jun. 2012.

[32] S. Khushalani, J. M. Solanki, and N. N. Schulz, "Optimized restoration of unbalanced distribution systems," IEEE Trans. Power Del., vol. 22, no. 2, pp. 624-630, May 2007.

[33] E. W. Dijkstra, "A note on two problems in connexion with graphs," Numerische Mathematik, vol. 1, no. 1, pp. 269-271, Dec. 1959.

[34] T. H. Cormen, C. Stein, R. L. Rivest, and C. E. Leiserson, Introduction to Algorithms, McGraw-Hill Higher Education, 2001.

[35] S. Ahmed, "A scenario decomposition algorithm for 0-1 stochastic programs," Oper. Res. Lett., vol. 41, no. 6, pp. 565-569, 2013.

[36] R. T. Rockafellar and R. J.-B. Wets, "Scenarios and policy aggregation in optimization under uncertainty," Math. of Oper. Res., vol. 16, no. 1, pp. 119-147, 1991.

[37] J.-P. Watson, D. L. Woodruff, and D. R. Strip, "Progressive hedging innovations for a stochastic spare parts support enterprise problem," Naval Research Logistics, 2007.

[38] J.-P. Watson and D. L. Woodruff, "Progressive hedging innovations for a class of stochastic mixed-integer resource allocation problems," Comput. Magag. Sci., vol. 8, no. 4, pp. 355-370, Nov. 2011.

[39] T. G. Crainic, X. Fu, M. Gendreau, W. Rei, and S. W. Wallace, "Progressive hedging-based meta-heuristics for stochastic network design," Networks, 58(2):114-124, 2011.

[40] IEEE PES Power System Analysis, Computing, and Economics Committee (Feb. 2014). IEEE 34 Node Test Feeder. [Online]. Available: http://ewh.ieee.org/soc/pes/dsacom/testfeeders/feeder34.zip

[41] IEEE PES Power System Analysis, Computing, and Economics Committee (Feb. 2014). IEEE 37 Node Test Feeder. [Online]. Available: http://ewh.ieee.org/soc/pes/dsacom/testfeeders/feeder37.zip

[42] S. Civanlar, J. J. Grainger, H. Yin, and S. S. H. Lee, "Distribution feeder reconfiguration for loss reduction," IEEE Trans. Power Del., vol. 3, no. 3, pp. 1217-1223, July 1988

[43] D. Das, "A fuzzy multiobjective approach for network reconfiguration of distribution systems," IEEE Trans. Power Del., vol. 21, no. 1, pp. 202-209, Jan. 2006. 


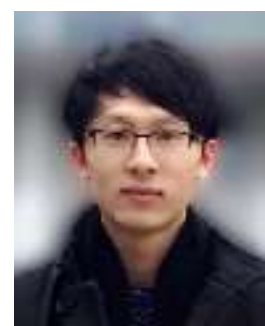

Shunbo Lei (S' 14) received his B.E. degree (2013) from Huazhong University of Science and Technology, Wuhan, China. Since 2013, he is a $\mathrm{Ph} . \mathrm{D}$. student with the University of Hong Kong, Hong Kong, China. He is currently visiting Argonne National Laboratory, Argonne, IL, USA. His research interests include optimization of power system operation, renewables integration and power system resilience.

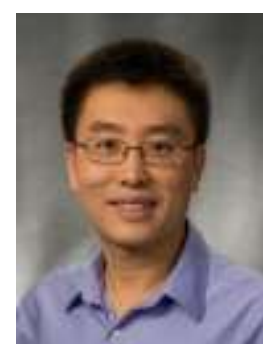

Jianhui Wang (M'07-SM'12) received the Ph.D. degree in electrical engineering from Illinois Institute of Technology, Chicago, IL, USA, in 2007.

Presently, he is the Section Lead for Advanced Power Grid Modeling at the Energy Systems Division at Argonne National Laboratory, Argonne, IL, USA.

Dr. Wang is the secretary of the IEEE Power \& Energy Society (PES) Power System Operations Committee. He is an associate editor of Journal of Energy Engineering and an editorial board member of Applied Energy. He is also an affiliate professor at Auburn University and an adjunct professor at University of Notre Dame. He has held visiting positions in Europe, Australia and Hong Kong including a VELUX Visiting Professorship at the Technical University of Denmark (DTU). Dr. Wang is the Editor-in-Chief of the IEEE Transactions on Smart Grid and an IEEE PES Distinguished Lecturer. He is also the recipient of the IEEE PES Power System Operation Committee Prize Paper Award in 2015.

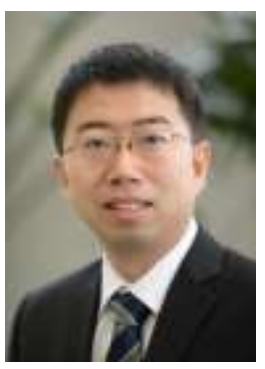

Chen Chen (M'13) received the B.S. and M.S. degrees in electrical engineering from Xi'an Jiaotong University, Xi'an, China, in 2006 and 2009 , respectively, and the Ph.D. degree in electrical engineering from Lehigh University, Bethlehem, PA, USA, in 2013. During 2013-2015, he worked as a Postdoctoral Researcher at the Energy Systems Division, Argonne National Laboratory, Argonne, IL, USA. Dr. Chen is currently a Computational Engineer with the Energy Systems Division at Argonne National Laboratory. His primary research is in optimization, communications and signal processing for smart electric power systems, cyber-physical system modeling for smart grids, and power system resilience.

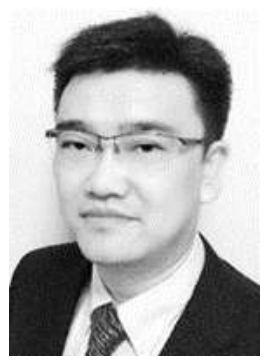

Yunhe Hou (M'08-SM'15) received the B.E. and $\mathrm{Ph} . \mathrm{D}$. degrees in electrical engineering from the Huazhong University of Science and Technology, Wuhan, China, in 1999 and 2005, respectively.

$\mathrm{He}$ was a Post-Doctoral Research Fellow at Tsinghua University, Beijing, China, from 2005 to 2007, and a Post-Doctoral Researcher at Iowa State University, Ames, IA, USA, and the University College Dublin, Dublin, Ireland, from 2008 to 2009. He was also a Visiting Scientist at the Laboratory for Information and Decision Systems, Massachusetts Institute of Technology, Cambridge, MA, USA, in 2010. He joined the faculty of the University of Hong Kong, Hong Kong, in 2009, where he is currently an Assistant Professor with the Department of Electrical and Electronic Engineering. 